\title{
Identification of UNC5A as a novel transcriptional target of tumor suppressor p53 and a regulator of apoptosis
}

\author{
YUJI MIYAMOTO ${ }^{1,2 *}$, MANABU FUTAMURA $^{1 *}$, NORIAKI KITAMURA ${ }^{1}$, \\ YASUYUKI NAKAMURA ${ }^{1}$, HIDEO BABA ${ }^{2}$ and HIROUFMI ARAKAWA ${ }^{1}$ \\ ${ }^{1}$ Cancer Medicine and Biophysics Division, National Cancer Center Research Institute, 5-1-1 Tsukiji, Chuo-ku, \\ Tokyo 104-0045; ${ }^{2}$ Department of Gastroenterological Surgery, Graduate School of Medical Science, \\ Kumamoto University, 1-1-1 Honjo, Kumamoto 860-8556, Japan
}

Received November 12, 2009; Accepted December 18, 2009

DOI: 10.3892/ijo_00000609

\begin{abstract}
UNC5A is an axon-guidance molecule, and plays a critical role in neuronal development and differentiation as a netrin-1 receptor. Emerging evidence suggests that axon guidance molecules including UNC5A regulate apoptosis in non-neuronal cells. Here, we report that UNC5A regulates apoptosis as a downstream target of p53. UNC5A expression was strongly induced by exogenous and endogenous p53. Chromatin immunoprecipitation (ChIP) revealed that $\mathrm{p} 53$ binds to a sequence in the promoter region of the UNC5A gene. Reporter assays showed that this sequence exhibits p53dependent transcriptional activity. Overexpression of UNC5A significantly suppressed colony formation of two glioblastoma cell lines-U373MG and T98G. UNC5A dramatically induced apoptosis through the activation of caspase-3 in various cancer cell lines, including LS174T (colon cancer), U373MG (glioblastoma), SH-SY5Y (neuroblastoma), and SKNAS (neuroblastoma). Finally, $\gamma$ irradiation strongly induced the expression of $U N C 5 A$ mRNA in the spleen and colon of $\mathrm{p} 53^{+/+}$mice, but not in those of $\mathrm{p} 53^{-/-}$mice, implying that the transcription of UNC5A in vivo is regulated by $\mathrm{p} 53$. These results suggest that UNC5A is a novel transcriptional target of p53 and plays a role in p53-dependent apoptosis.
\end{abstract}

\section{Introduction}

The p53 gene is mutated in $>50 \%$ of human cancers. The p53 protein activates transcription of many downstream target genes by binding to the regulatory sequences of these genes, thereby increasing their expression levels and enhancing their

Correspondence to: Dr Hirofumi Arakawa, Cancer Medicine and Biophysics Division, National Cancer Center Research Institute, 5-1-1-Tsukiji, Chuo-ku, Tokyo 104-0045, Japan

E-mail: harakawa@ncc.go.jp

${ }^{*}$ Contributed equally

Key words: p53, dependence receptor, UNC5A, apoptosis, axon guidance functional activities in response to various types of cellular stress including DNA damage. A considerable number of p53 target genes have been identified so far $(1,2)$. The core functions of p53 are cell cycle arrest, apoptosis, DNA repair, and antiangiogenesis, which are mediated by the p53 targets p21/WAF1, p53AIP1, p53R2, and SEMA3F, respectively. By regulating these target genes, p53 prevents the damaged cell from malignant transformation and serves as a guardian of the genome (3). In fact, p53 induces cell cycle arrest via p21/WAF1 in order to repair damaged DNA, whereas it induces apoptosis via p53 upregulated modulator of apoptosis (PUMA) and Noxa in order to eliminate the potential cancer cells containing severely damaged DNA. Therefore, identification of apoptosis-inducing target genes of p53 is very important to clarify the mechanism of p53-regulated tumor suppression.

The deleted in colorectal cancer $(D C C)$ gene and the UNC5 gene family members act as receptors of netrin-1 $(4,5)$. The $U N C 5$ gene family consists of four related genes including $U N C 5 A, U N C 5 B, U N C 5 C$, and UNC5D. The UNC5s encode type-I transmembrane proteins. Rodent $U N C 5 H 1, U N C 5 H 2$, $U N C 5 H 3$, and $U N C 5 H 4$ are orthologs of human $U N C 5 A$, $U N C 5 B, U N C 5 C$, and $U N C 5 D$, respectively. DCC and UNC5s play an essential role in axon guidance during neuronal development and differentiation; in addition, they regulate apoptosis as 'dependence receptors' $(6,7)$. In the absence of netrin-1, DCC and UNC5s transmit apoptotic signals. However, in the presence of netrin-1, the survival signal is activated via the interaction of netrin- 1 and these receptors $(6,7)$. Thus, these receptors positively and negatively regulate apoptosis in a netrin-1-dependent manner.

Although the UNC5 gene family is primarily important for axon guidance in neuronal cells, it is associated with tumorigenesis in non-neuronal cells $(8,9)$. For example, the expression of $U N C 5 A, U N C 5 B$, and $U N C 5 C$ is frequently downregulated in human colorectal, stomach, breast, and lung cancers $(10,11)$. This downregulation is associated with the genomic deletion of the respective genes. Furthermore, the netrin-1 receptors, including UNC5H1, UNC5H2, and DCC, have been shown to induce apoptosis in non-neuronal cells (6-14). UNC5B (also termed p53RDL1) mediates p53dependent apoptosis as a p53-target gene (15). UNC5D was 
recently identified as a p53 target gene, whose gene product was found to be involved in the p53-dependent apoptotic response induced after DNA damage (16).

These results prompted us to examine whether UNC5A may be directly regulated by the tumor suppressor p53 and be involved in p53-dependent apoptosis. We propose that UNC5A is a novel p53 target gene and that it regulates apoptosis.

\section{Materials and methods}

Cell lines. LS174T (colon cancer), H1299 (lung cancer), HepG2 (hepatoblastoma), U373MG (glioblastoma), T98G (glioblastoma), SH-SY5Y (neuroblastoma) and SKNAS (neuroblastoma) were purchased from American Type Culture Collection (ATCC; Manassas, VA, USA). These cell lines were maintained under the conditions recommended by the depositor.

RNA interference. By using LS174T containing wild-type p53 (wt-p53), we established the p53-knock-down cell lines (LS174T-p53-KD) and the control cell lines (LS174T-Cont) as previously described (17). In brief, LS174T cells were infected with SI-MSCV-puro-H1R-p53Ri retrovirus for downregulation of p53 expression and with SI-MSCV-puro-H1R retrovirus for negative control. Then the infected cells were selected with $1 \mu \mathrm{g} / \mathrm{ml}$ puromycin for 2 weeks, and the single clones were isolated.

Reverse transcription-polymerase chain reaction ( $R T-P C R)$ analysis. HepG2 and H1299 cells were infected with Ad-wtp53, Ad-p53-46F or Ad-EGFP at 30 multiplicity of infection (MOI) $(17,18)$. Using TRIzol reagent (Invitrogen, Carlsbad, CA, USA) total RNAs were isolated from the cells collected at the time-points of $0,6,12,24$, and $48 \mathrm{~h}$ after infection, respectively. LS174T-p53-KD and LS174T-Cont cell lines were treated with $1 \mu \mathrm{g} / \mathrm{ml}$ of adriamycin (doxorubicin) for $2 \mathrm{~h}$, and total RNAs were also obtained at the same time-points after the treatment. A 3- $\mu$ g aliquot of each total RNA was reverse-transcribed and the PCR was run in the exponential region (18-35 cycles) to allow semi-quantitative comparisons among cDNAs developed from identical reactions. In order to compare the expression level of UNC5 family, pcDNA 3.1UNC5A-sense/antisense, pcDNA 3.1-UNC5B-sense/antisense were transfected to H1299 cells, and Ad-UNC5A, Ad-UNC5B, Ad-UNC5D were infected to U373MG cells at an indicated MOI, respectively. Twenty-four hours later, the cells were harvested and subjected to semi-quantitative RT-PCR as described above.

Western blot analysis. Adriamycin-treated cells were lysed in Radio-immuno-protein-assay (RIPA) buffer (150 mM NaCl, $50 \mathrm{mM}$ Tris- $\mathrm{HCl} \mathrm{pH} 8.0,0.1 \%$ sodium dodecyl sulfate (SDS), $1 \%$ Nonidet P (NP)-40, protease inhibitor cocktail). Aliquots $(15 \mu \mathrm{g})$ of the soluble proteins were loaded on SDS-polyacrylamide gel and transferred to Hybond-P membranes (GE Healthcare Bio-Sciences, Piscataway, NJ, USA). After blocking, the membranes were incubated with anti-p53 (Ab-6, 1:1000) (Merck, Darmstadt, Germany) or anti- 3 -actin antibody (AC-74, 1:5000) (Sigma, St. Louis, MO, USA) then hybridized with horseradish peroxidase (HRP)-conjugated secondary antibody, and detected by ECL Western blotting detection reagents (GE Healthcare).

ChIP assay. ChIP assay was performed using the chip assay kit (Upstate, Lake Placid, NY, USA) as recommended by the manufacturer. In brief, H1299 cells plated on a 10-cm-dish $\left(2 \times 10^{6}\right)$ were infected with either Ad-p53 or Ad-EGFP at $30 \mathrm{MOI}$. After $24 \mathrm{~h}$, genomic DNA and protein were crosslinked by adding formaldehyde ( $1 \%$ final concentration) directly into culture medium and incubated for $15 \mathrm{~min}$ at $37^{\circ} \mathrm{C}$. Cells were lysed in $200 \mu 1$ of SDS lysis buffer with a protease inhibitor cocktail and sonicated to generate 200-1000 bp-long DNA fragments. After centrifugation, cleared supernatant was diluted 10-fold with SDS lysis buffer and incubated at $4^{\circ} \mathrm{C}$ overnight with the specific antibody. Immune complexes were precipitated, washed, and eluted by elution buffer $(1 \%$ SDS, $1 \mathrm{mM} \mathrm{NaHCO}$ ). The eluted DNA-protein complexes were reversed by heating at $65^{\circ} \mathrm{C}$ for $5 \mathrm{~h}$. The DNA was phenol-extracted, ethanol-precipitated and re-suspended in $50 \mathrm{ml}$ of TE buffer then subjected for PCR amplification with 30 cycles.

Gene reporter assay. DNA fragments including potential p53-binding sequence (p53BS) of UNC5A were amplified by PCR and cloned into pGL3-promoter vector (Promega, Madison, WI, USA). The reporter plasmid was co-transfected into H1299 with either wild-type or mutant (R175H) p53 expression vector in combination with pRL-CMV vector (Promega). Twenty-four hours later, the cells were lysed and subjected to measurements of luciferase activity. pGL3 vector with p53BS for $p 21$ was used for positive control. In addition four constructs expressing p53 family gene $(p 73 \alpha, p 73 \beta, p 63 \alpha, p 63 \gamma)(19,20)$ were also used as described previously (21).

Plasmid construction. The entire coding region of UNC5A and $U N C 5 B$ amplified by RT-PCR was cloned into pCRBlunt II-TOPO vector (Invitrogen) and sequenced. The fragment containing the whole UNC $5 A$ or $U N C 5 B$ sequence were digested by EcoRI and cloned into pcDNA3.1 (+) (Invitrogen) to prepare sense-strand UNC5A or UNC5B (pcDNA-UNC5A-S or pcDNA-UNC5B-S) and antisense-strand UNC5A or UNC5B (pcDNA-UNC5A-AS or pcDNA-UNC5B-AS) for colony formation assay.

Colony formation assay. Five microgram of pcDNA3.1UNC5A-S, UNC5B-S, -UNC5A-AS, UNC5B-AS, and empty pcDNA3.1 (+) vector were transfected into the U373MG or T98G $\left(1.5 \times 10^{6} / 10 \mathrm{~cm}\right.$ dish $)$ cells. Twenty-four hours later, the transfected cells were diluted, replated onto $10 \mathrm{~cm}$ dishes and cultured in G418-containing media $(1 \mu \mathrm{g} / \mathrm{ml})$. Two weeks later, the drug-resistant colonies were fixed with $10 \%$ formalin then stained with crystal violet. Colonies of $>1 \mathrm{~mm}$ diameter were counted.

Adenovirus construction. Adenovirus (Ad) expressing wt-p53, p53-46F, enhanced green fluorescent protein (EGFP), and UNC5B were prepared as described previously $(18,21)$. Ad-UNC5A and Ad-UNC5D were also generated and purified. In brief, blunt-ended full-length UNC5A and UNC5D were 
A

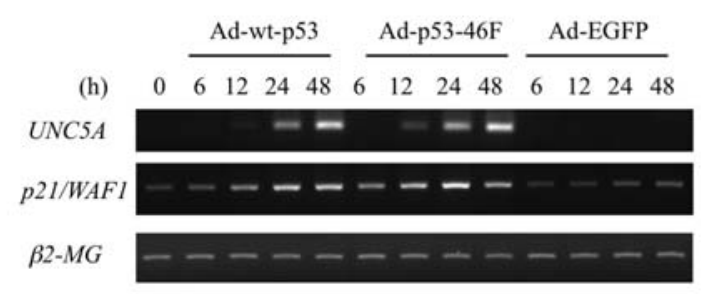

(H1299)

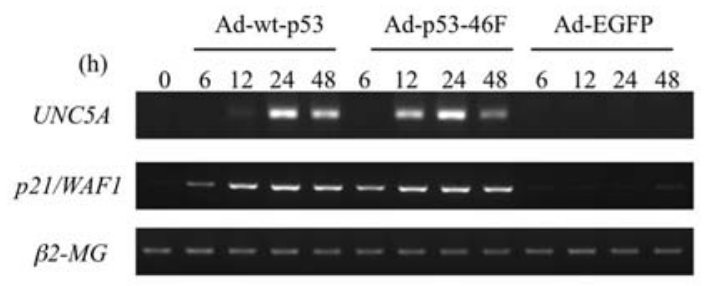

B

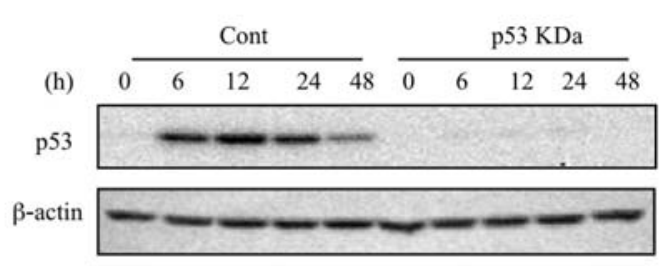

C

(LS174T)

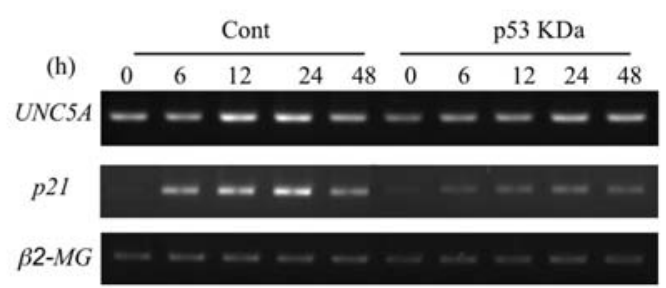

Figure 1. UNC5A is a p53-inducible gene. (A) Induction of UNC5A by exogenous p53. The expressions of UNC5A mRNAs are shown by RT-PCR in HepG2 and H1299 cells infected with either Ad-p53-wt, Ad-p53-46F or Ad-EGFP at the indicated times. $\beta 2-M G$ (microglobulin) was used as a loading control. (B) Expression level of endogenous p53 after DNA damage. (C) Induction of UNC5A by endogenous p53. The expression levels of p53 protein and UNC5A mRNA are shown by Western blot analysis and RT-PCR, respectively, in LS174T control and LS174T-p53-KD cells treated with $1 \mu \mathrm{g} / \mathrm{ml}$ of adriamycin for $2 \mathrm{~h}$ at the indicated times. p21 is a positive control. B-actin and B2-MG are used as a loading control.

also cloned into the SmiI site of the cosmid pAxCAwtit (Takara, Otsu, Japan), which contains the CAG promoter and the entire genome of type 5 adenovirus except for E1 and E3 regions, then transfected to HEK293 (human embryonic kidney cell). Viruses were propagated in the 293 cells and purified as described previously (18). Expression levels of UNC5A UNC5B, or UNC5D were evaluated by RT-PCR at $24 \mathrm{~h}$ post infection to U373MG cells at the indicated MOI.

Apoptosis assay. U373MG, LS174T, SH-SY5Y, and SKNAS cells were seeded on 6 well-plate and infected with either Ad-UNC5A, Ad-UNC5B, Ad-UNC5D or Ad-EGFP at indicated MOI. After $72 \mathrm{~h}$ of infection, the cells were collected and fixed with $70 \%$ ethanol then treated with $10 \mu \mathrm{g} / \mathrm{ml}$ of RNase A for $30 \mathrm{~min}$ at $37^{\circ} \mathrm{C}$. After centrifugation the cells were stained with $50 \mu \mathrm{g} / \mathrm{ml}$ of propidium iodine and subjected to flow cytometry analysis by FACS Calibur (BecktonDickinson, Franklin Lakes, NJ, USA). Next $20 \mu \mathrm{M}$ of ZVAD-FMK (MBL, Nagoya, Japan), a pan-caspase inhibitor was added to examine whether UNC5A-inducing cell death was caspase-dependent.

Caspase-3 activity. Caspase-3 activity was measured using tetrapeptide p-nitroanilide (pNA) substrate (Ac-DEVD-pNA, Sigma) in a colorimetric assay. After $48 \mathrm{~h}$ of Ad-UNC5 infection at $60 \mathrm{MOI}$ to LS174T, the cells were lysed in cell lysis buffer (50 mM HEPES pH 7.4, $100 \mathrm{mM} \mathrm{NaCl}, 0.1 \%$ CHAPS, 1 mM EDTA, $10 \%$ glycerol, $0.1 \%$ NP-40). The assay was performed in 96-well plates by incubating $10 \mu \mathrm{l}$ of cell lysate with $90 \mu 1$ of reaction buffer (50 mM HEPES $\mathrm{pH} 7.4,100 \mathrm{mM} \mathrm{NaCl}, 0.1 \%$ CHAPS, 1 mM EDTA, $10 \%$ glycerol) containing $250 \mu \mathrm{M}$ of Ac-DEVD-pNA for caspase-3 (Sigma) at $37^{\circ} \mathrm{C}$ for $4 \mathrm{~h}$. Then the samples were read in a spectrophotometer at $405 \mathrm{~nm}$. p53 knockout mice. p53-deficient mice were a gift from Dr S. Aizawa, Center for Developmental Biology, RIKEN, Japan (22). $\mathrm{p} 53^{+/+}$and $\mathrm{p} 53^{-/-}$mice were irradiated by $10 \mathrm{~Gy}$. Then the mice were euthanized and harvested at indicated times after irradiation. The indicated organs were isolated and total RNAs were purified from the tissues, from which cDNAs were synthesized and subjected to RT-PCR analysis. All mouse procedures were carried out according to the recommendations of the Institutional Animal Care and Use Committee of the National Cancer Center at Tsukiji, Japan.

\section{Results}

Identification of UNC5A as a novel p53-inducible gene. Since $U N C 5 B$ (p53RDL1) and UNC5D were reported to be $\mathrm{p} 53$ target genes $(15,16)$, we hypothesized that other $U N C 5 s$, including $U N C 5 A$, may also be regulated by tumor suppressor p53. Therefore, we determined the p53-dependent inducibility of UNC5A by using HepG2 and H1299 cells. The expression of $U N C 5 A$ was induced by adenoviruses expressing exogenous p53 (Ad-wt-p53 and Ad-p53-46F) $(17,18)$, but not by the control adenovirus expressing enhanced green fluorescent protein (Ad-EGFP) (Fig. 1A). To further confirm the induction of UNC5A by endogenous p53, we generated a p53-knockdown (p53 kDa) cell line by using the colorectal cancer cell line LS174T. The expression of endogenous p53 increased in LS174T-Cont cells after adriamycin treatment at a concentration of $1 \mu \mathrm{g} / \mathrm{ml}$ for $2 \mathrm{~h}$, whereas negligible $\mathrm{p} 53$ expression was detected in LS174T-p53-KD cells after this treatment (Fig. 1B). Consistent with the p53 expression, the expression of UNC5A mRNA increased in LS174T-Cont cells, but not in LS174T-p53-KD cells, after adriamycin treatment; this expression profile was in accordance with that of $p 21 / W A F 1$, which was used as a positive control (Fig. 1C). These results 
A

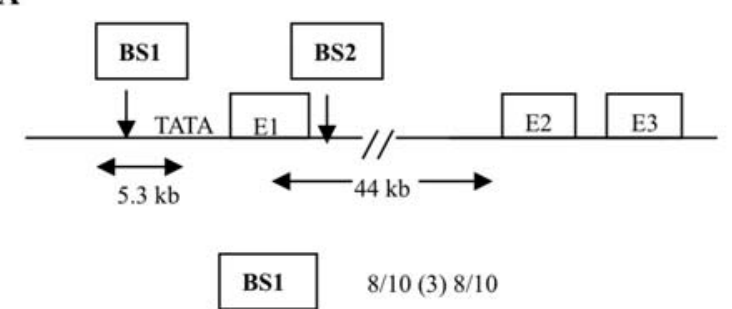

GGGC TT G aaC aat GAAC TA G ga T

| | | | | | | | | | | |

RRRCWWGYYY RRRCWWGYYY

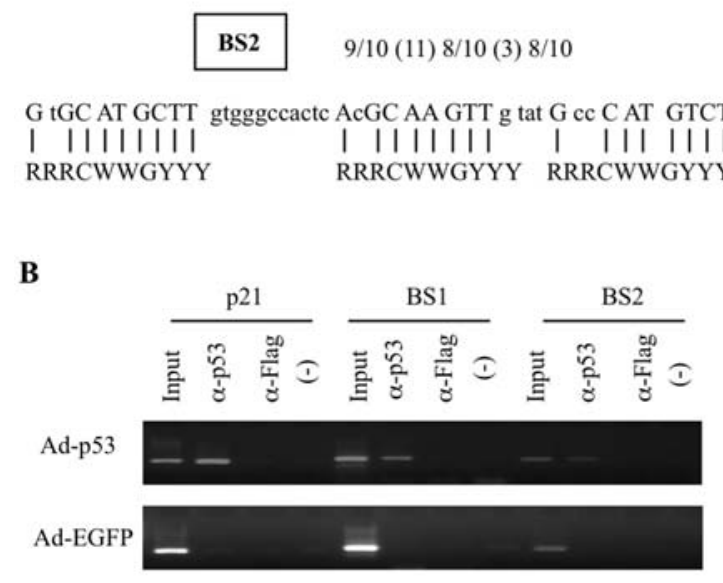

C
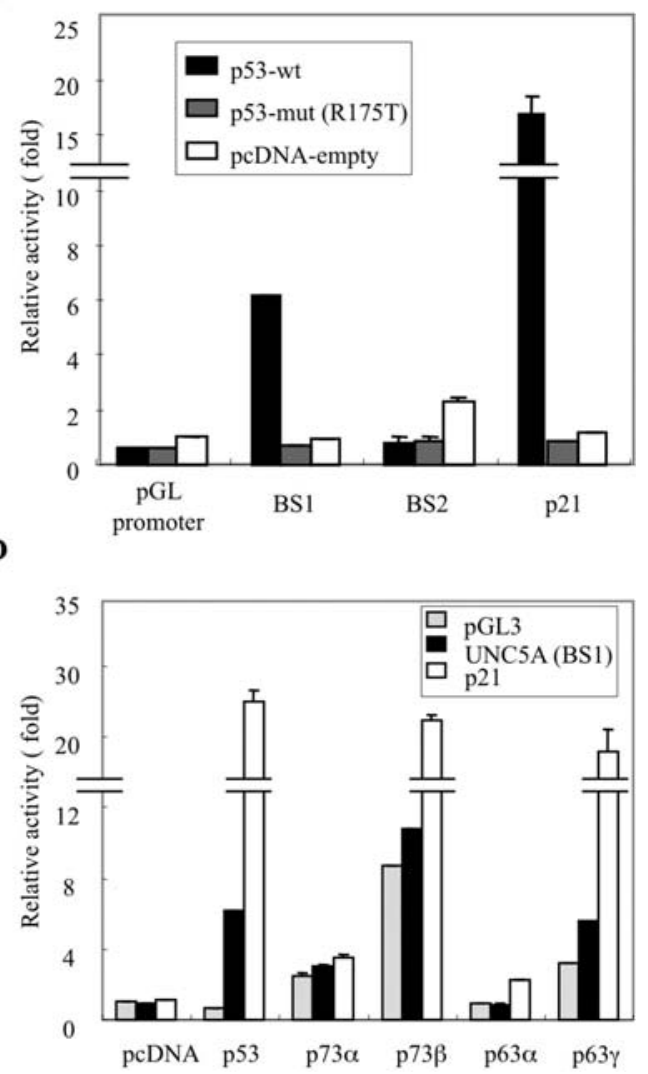

Figure 2. UNC5A is a direct target gene of p53. (A) Identification of a potential p53-binding sequence (p53BS) in the genomic DNA of UNC5A. Two candidate sequences (BS1 in the promoter region and BS2 in intron 1) were found. The sequences are shown. E1, E2, and E3 indicate the exons. TATA indicates the TATA box sequence. (B) ChIP assay. The cross-linked DNA-protein complex was immunoprecipitated by anti-p53 antibody ( $\alpha$-p53), followed by PCR amplification. Immunoprecipitates by anti-Flag ( $\alpha$-Flag) or in the absence of antibody (-) served as negative controls. Input chromatin (Input) represents a part of sonicated chromatin before immunoprecipitation. Promoter sequence of p21 was used as a positive control. (C) Luciferase assay. Reporter plasmid containing BS1 or BS2 was cotransfected with p53-wt, p53-mut (R175H) or empty expression vector into H1299 cells. Relative luciferase activities are shown. (D) Luciferase assay. Reporter plasmid containing BS1 was cotransfected with expression vectors of the p53 family genes such as $73 \alpha, p 73 \beta$, p $63 \alpha$ and $\mathrm{p} 63 \gamma$. Relative luciferase activities are shown.

suggest that the transcription of $U N C 5 A$ is activated by both exogenous and endogenous p53.

UNC5A is a direct transcriptional target of p53. To further investigate whether the expression of UNC5A is directly regulated by $\mathrm{p} 53$, we searched for putative p53-binding sequence(s) (p53BS) in the UNC5A genome. We eventually found two potential sequences, one located in the 5'-upstream region (BS1), and the other, in intron 1 (BS2) (Fig. 2A). We performed chromatin immunoprecipitation (ChIP) to examine whether p53 binds to these sequences. The DNA fragments precipitated by the anti-p53 antibody in H1299 cells infected with Ad-p53 were shown to contain both BS1 and BS2 (Fig. 2B), thereby indicating that both BS1 and BS2 could interact with $\mathrm{p} 53$.

To determine whether these sequences actually exhibit p53-dependent transcriptional activity, we performed reporter assays. DNA fragments were amplified by polymerase chain reaction (PCR), cloned into a pGL-promoter vector (pGL-BS1 or pGL-BS2), and cotransfected with wild-type or mutant p53 expression vectors. The luciferase activity was enhanced only when pGL-BS1 was cotransfected with the wild-type p53 expression vector (Fig. 2C), indicating that BS1 exhibits p53-dependent transcriptional activity.
We examined whether the transcription of $U N C 5 A$ could be activated by other p53 family genes, namely, p63 and p73. However, unlike p53, neither p63 nor p73 could significantly enhance luciferase activity (Fig. 2D); these results suggest that UNC5A transcription is specifically activated by $\mathrm{p} 53$. Therefore, we concluded that UNC5A is a bona fide target of p53.

UNC5A plays a role in the suppression of cell growth. To investigate the role of UNC5A in the regulation of cell growth, we carried out a colony formation assay. Before the assay, we investigated the expression levels of sense/antisenseUNC5A and sense/antisense-UNC5B mRNAs derived from the plasmids designed to express them. We confirmed that the mRNA levels of sense-UNC5A and sense- $U N C 5 B$ were very similar (Fig. 3A). U373MG and T98G cells transfected with plasmids expressing sense-UNC5A showed a significant decrease in colony number when compared with those transfected with plasmids expressing antisense-UNC5A (Fig. 3B). Moreover, the colony number of sense-UNC5A-tranfected cells was equivalent to that of sense-UNC5B-transfected cells (Fig. 3B). Since UNC5B/p53RDL1 is known to induce apoptosis (15), we strongly suspected that UNC5A may possess apoptotic activity. 
A

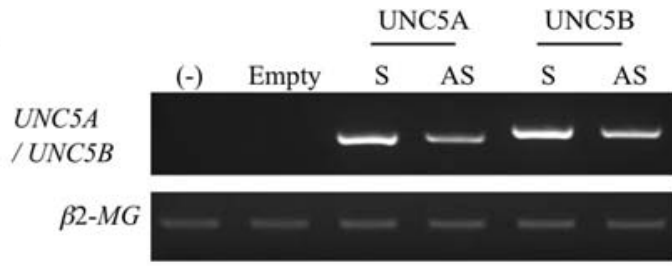

B

(U373MG)

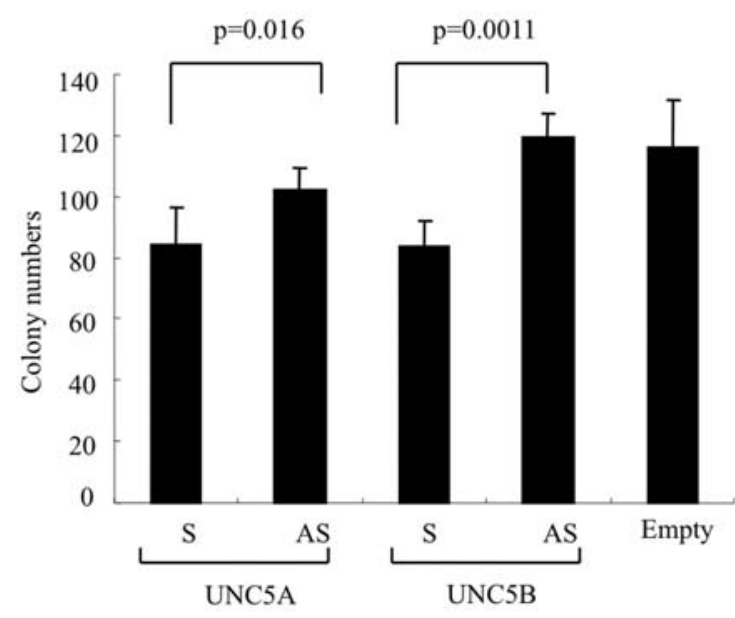

(T98G)

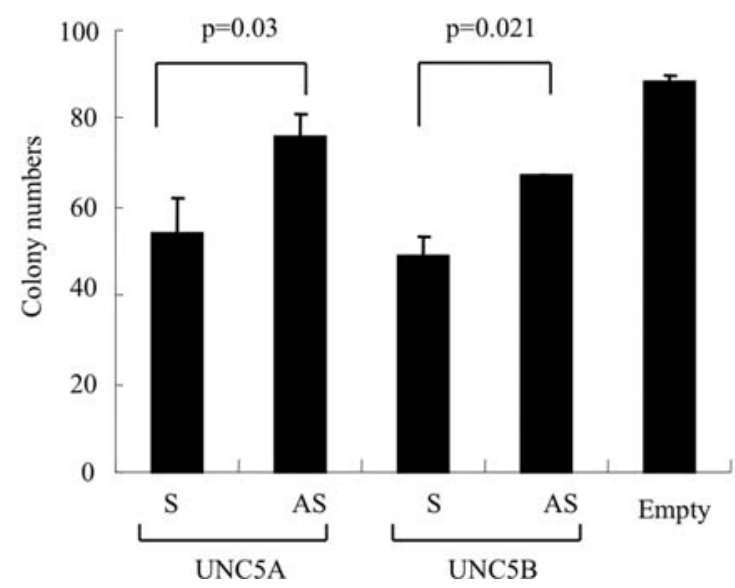

Figure 3. UNC5A induces cell growth suppression. (A) Expression levels of UNC5A and UNC5B mRNAs in H1299 cells. Twenty-four hours after transfection of pcDNA3.1 sense/antisense-UNC5A, pcDNA3.1 sense/ antisense-UNC5B or pcDNA 3.1 (empty), RT-PCR was performed with the UNC5A or UNC5B specific primers. $\beta 2-M G$ was used as a quality control. (B) Colony formation assay. The number of G418-resistant colonies derived from U373MG or T98G cells transfected with the plasmids are shown. The experiments were performed three times, and the average score is indicated with error bars of standard division. ${ }^{*} \mathrm{p}<0.05$ was considered statistically significant.

UNC5A induces apoptosis. To evaluate the role of UNC5A in apoptosis, we prepared adenoviral vectors designed to express UNC5A, UNC5B, or UNC5D. UNC5B and UNC5D have been shown to induce apoptosis $(15,16)$. We confirmed that $U N C 5 A, U N C 5 B$, and $U N C 5 D$ mRNAs were well expressed in cells infected with Ad-UNC5A, Ad-UNC5B, and Ad-UNC5D, respectively, in a dose-dependent manner (Fig. 4A). Four cancer cell lines-LS174T (colon cancer), U373MG (glioblastoma), SH-SY5Y (neuroblastoma), and
$\mathbf{A}$

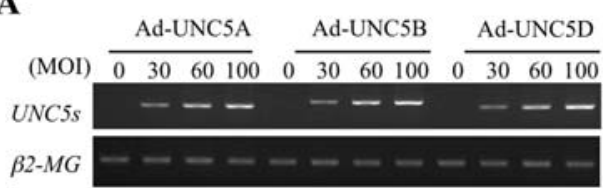

B

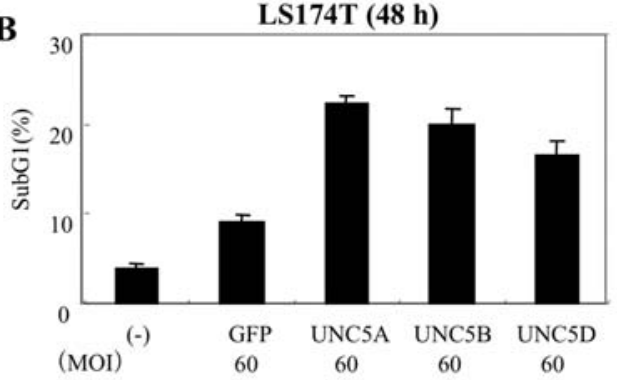

SH-SY5Y (72 h)

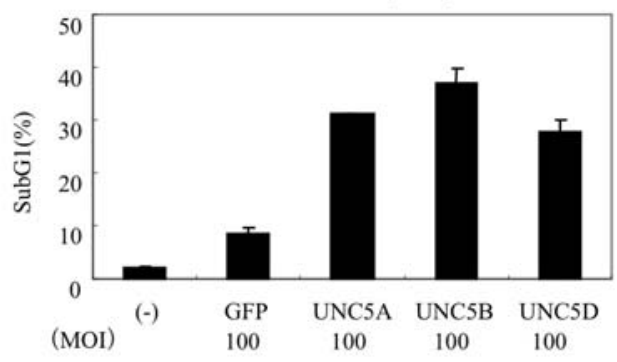

U373MG (72 h)

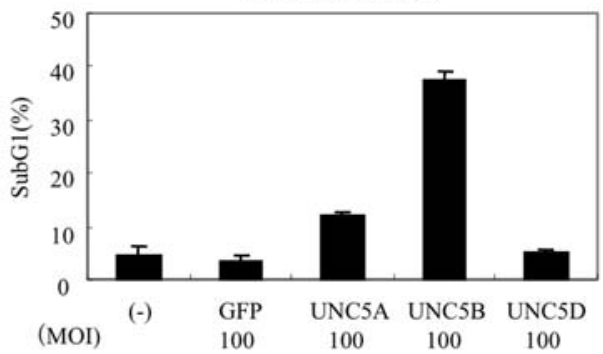

SKNAS (72 h)

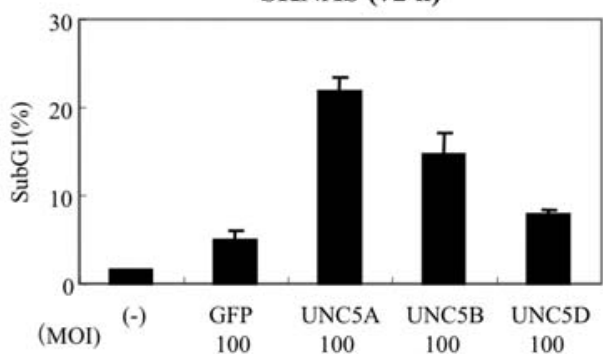

Figure 4. UNC5A induces apoptosis. (A) Expression levels of UNC5A, UNC5B and UNC5D mRNAs. The expressions of UNC5A, UNC5B and UNC5D mRNAs in U373MG cells $24 \mathrm{~h}$ after infection with Ad-UNC5A, Ad-UNC5B and Ad-UNC5D at indicated MOIs. $\beta 2-M G$ was used as a quality control. (B) Apoptosis assay. Apoptotic cells were evaluated by FACS Scan analysis. The average values of apoptotic cells are shown with error bars of standard division. All experiments were repeated independently three times and representative results are shown.

SKNAS (neuroblastoma) were infected with Ad-UNC5A, Ad-UNC5B, or Ad-UNC5D at various multiplicities of infection (MOIs), and apoptotic cells were analyzed by fluorescence-activated cell sorting (FACS) at the indicated times. UNC5A, like UNC5B, was able to strongly induce apoptosis in all cancer cell lines (Fig. 4B), particularly LS174T, 
A

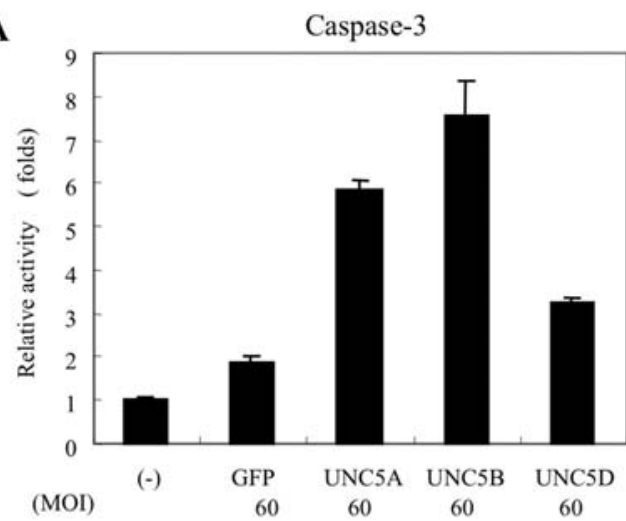

B

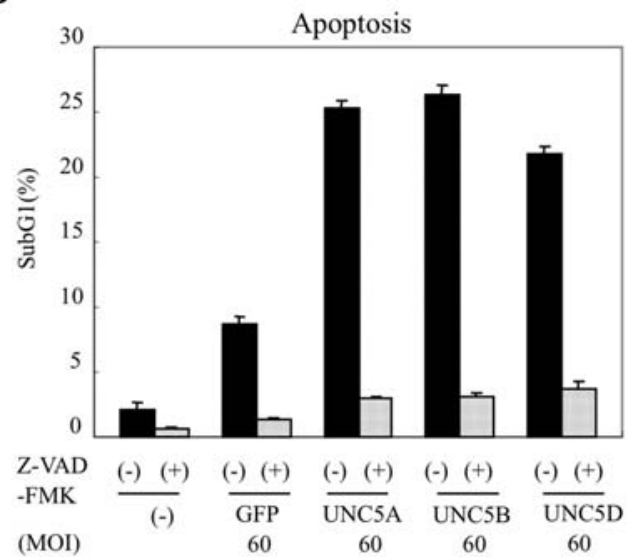

Figure 5. UNC5A regulates apoptosis in a caspase-dependent manner. (A) Caspase-3 activity. Forty-eight hours after infection with the indicated adenovirus vectors, the activity of caspase-3 in LS174T cells was measured with Ac-DEVD-pNA. Relative activities were indicated with error bars of standard division compared to control cells (-). (B) Apoptosis assay with Z-VAD-FMK. LS174T cells were treated with $50 \mu \mathrm{M}$ of caspase inhibitor Z-VAD-FMK before infection. Forty-eight hours after infection, apoptotic cells were evaluated by FACS Scan analysis. The average values of apoptotic cells are shown with error bars of standard division.

SH-SY5Y, and SKN-AS. However, in U373MG cells, UNC5A induced apoptosis to a much lesser extent than UNC5B. Interestingly, UNC5D was unable to induce apoptosis in U373MG cells, suggesting that the role of UNC5D in apoptosis is tissue specific.

To further confirm the role of UNC5A in apoptosis, we investigated whether UNC5A activates caspase-3. UNC5A, like UNC5B, was able to dramatically activate caspase-3. In addition, the caspase-inhibitor Z-VAD-FMK completely inhibited the DNA fragmentation induced by UNC5A (Fig. 5B), suggesting that UNC5A-induced cell death is caspase dependent. These results clearly suggest that UNC5A induces apoptosis via the activation of caspase- 3 and that UNC5A is a mediator of p53-dependent apoptosis.

UNC5A functions as an in vivo mediator of p53 response after DNA damage. To assess whether UNC5A truly functions as an in vivo mediator of p53 response, we determined the p53-dependent inducibility of UNC5A by using p53-knockout mice. We irradiated $\mathrm{p} 53^{+/+}$and $\mathrm{p} 53^{-/}$mice with 10 Gy of $\gamma$ rays and isolated RNA from the spleen, colon, brain, and thymus at the indicated times. Interestingly, we observed strong induction of UNC5HI (mouse UNC5A) in the spleen and colon (Fig. 6) and slight induction in the thymus in response to DNA damage; the induction was p53-dependent in the case of all three organs. Furthermore, $U N C 5 H 2$ (mouse $U N C 5 B$ ) and $U N C 5 H 4$ (mouse $U N C 5 D$ ) were induced by p53 in the spleen, colon, and thymus in response to DNA damage. However, none of the three UNC5s showed any changes in mRNA levels in the brain after DNA damage. These results suggest that UNC5s, including UNC5A, play a critical role in the p53-dependent apoptosis induced after DNA damage in vivo, especially in non-neuronal tissues.

\section{Discussion}

As 'dependence receptors', axon guidance molecules - the UNC5s - are known to regulate apoptosis in non-neuronal tissues and to be associated with tumor suppression. For example, the dependence receptor UNC5B mediates p53dependent apoptosis by activating caspase-3, whereas apoptosis is inhibited when its ligand netrin- 1 binds to UNC5B (15). UNC5B is a target gene of p53. Interestingly, netrin- 1 is also a target of p53 $(8,9)$. Thus, p53 could regulate both the ligand and the receptor for control of cell death and survival; however, the mechanism by which this regulation occurs still remains unclear. Llambi et al showed that rat $\mathrm{UNC} 5 \mathrm{H} 1, \mathrm{H} 2$, and $\mathrm{H} 3$ are involved in caspase-dependent apoptosis through their cleavage at putative caspase cleavage sites in the intracellular domains (12). The newly identified $U N C 5 D$ was also shown to be a target of p53 and to regulate apoptosis in response to DNA damage (16). These results prompted us to examine the relationship between human UNC5A and $\mathrm{p} 53$. We identified a p53-binding site (p53BS) in the 5'-upstream region of the UNC5A gene, which was found to functionally interact with the p53 protein in ChIP and reporter assays. The p53-binding site of UNC5A was evidently activated by p53, but not by other 53 family proteins, thereby suggesting that p53 plays a central role in the apoptotic pathway mediated by dependence receptors.

We showed that $U N C 5 A$ is induced in vivo by using irradiated $\mathrm{p} 53^{+/+}$or $\mathrm{p} 53^{-/}$mice (Fig. 6). Like the other UNC5 genes, UNC5A was particularly induced in the spleen and colon after DNA damage. Although UNC5A expression is usually high in the brain, it did not show an increase after DNA damage. UNC5A plays an important role in neuronal apoptosis independent of netrin-1 (23); this suggests that UNC5A may function independent of p53 in neuronal tissues. Therefore, we believe that UNC5A may play a critical role in p53-dependent apoptosis in non-neuronal tissues, in response to DNA damage.

The UNC5s share several domains, such as an immunoglobulin domain, a TSP1 (thrombospondin 1) domain, a ZU-5 domain (a homology domain found in zona occludens protein-1 and UNC5 proteins), a death domain at the $\mathrm{C}$ terminal region $(5,12)$, and the classical caspase cleavage site downstream of the transmembrane region (DXXD) (24). Human UNC5A also possesses a putative caspase cleavage site (337DVAD340) at the $\mathrm{N}$-terminus of the intracellular domain. In fact, UNC5A and UNC5B showed similar levels of growth suppression or apoptosis induction via caspase-3 activation. Since apoptosis induced by UNC5s was almost completely suppressed by a pan-caspase inhibitor, we think that UNC5A 
(Spleen)

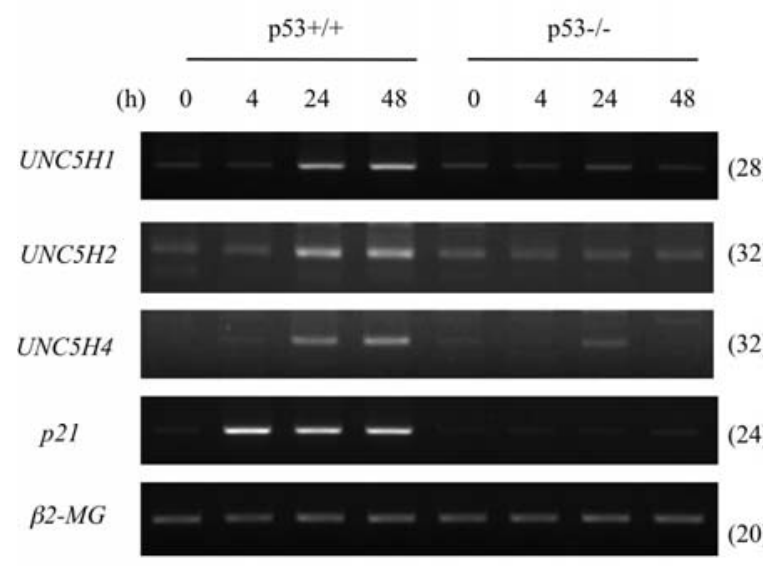

(Colon)

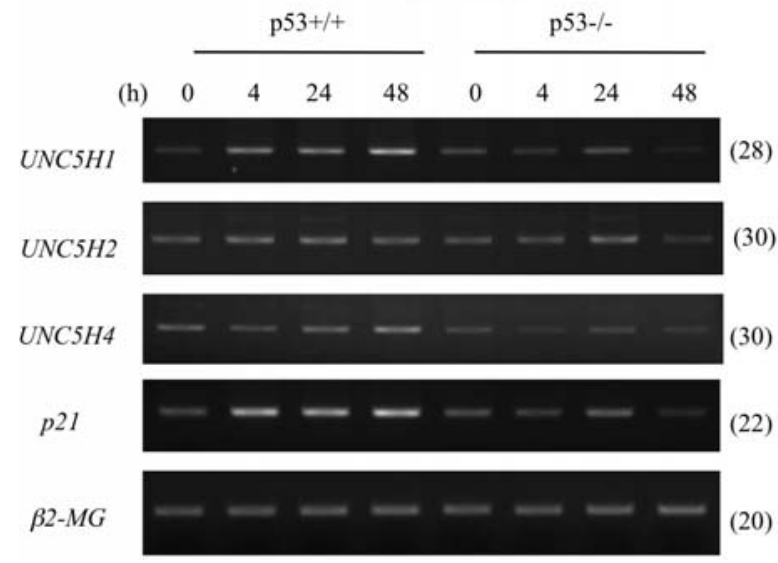

(Brain)

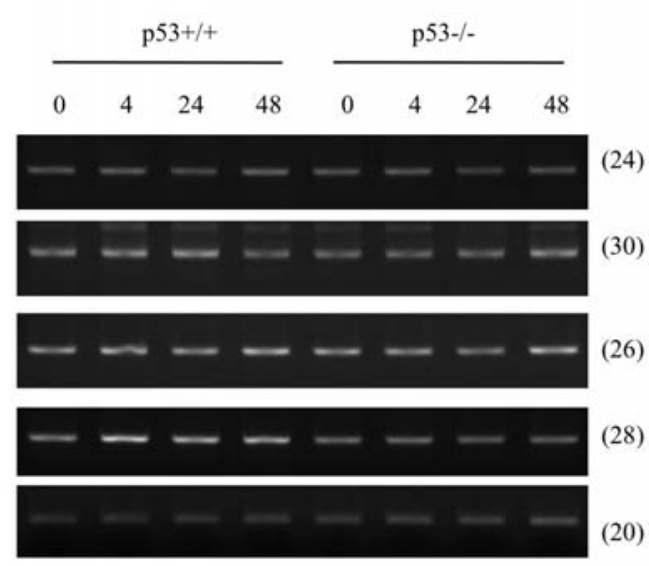

(Thymus)

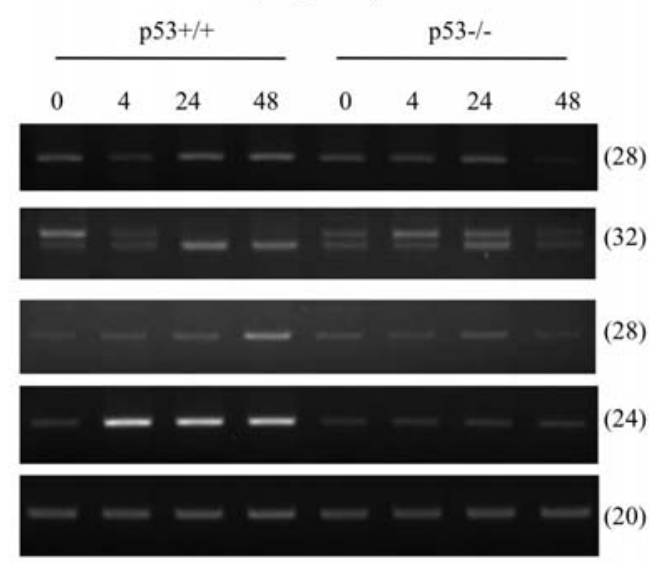

Figure 6. Potential in vivo role of UNC5A in p53-regulated response to DNA damage. RT-PCR results indicated expression levels of UNC5H1, UNC5H2, UNC5H4, and p 21 mRNA in the spleen, brain, colon and thymus tissues. Each tissue was isolated from p53 $3^{+/+}$and $\mathrm{p} 53^{-/-}$mice $24 \mathrm{~h}$ after irradiation with $\gamma$-ray $(10 \mathrm{~Gy})$. The number of cycles for each RT-PCR is indicated in parentheses. $\beta 2-M G$ was used as a loading control.

is probably involved in caspase-dependent apoptosis and that there is most likely a common mechanism for UNC5-induced apoptosis.

All three UNC5s strongly induced apoptosis in the LS174T and SH-SY5Y cells, which contain wild-type p53. However, in the SKNAS and U373MG cells, which contain mutant p53, the ability of the three UNC5s to induce apoptosis differed. For example, UNC5B was able to strongly induce apoptosis whereas UNC5A and UNC5D were not. In particular, UNC5D revealed weaker ability to induce apoptosis in SKNAS and U373MG cells, both of which contain mutant p53. These results are consistent with those reported by Wang et al, where UNC5D was found to amplify the p53dependent apoptotic response (16). We propose that apoptosis induced by UNC5s may depend on not only the cell type but also the p53 status. The p53 status probably affects UNC5Aor UNC5D-induced apoptosis. Further studies will enable a better understanding of the precise role of UNC5s as dependence receptors and in $\mathrm{p} 53$-dependent apoptosis.

\section{Acknowledgements}

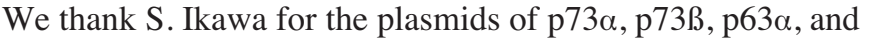
p63 $\gamma$, A. Nakagawara for the plasmid of UNC5D, S. Aizawa for p53-deficient mice. We also thank S. Usuda, and I. Hyo for their technical assistance. This work was supported in part by grants (H.A.) from the Ministry of Health, Labor and Welfare, Japan, and the Ministry of Education, Culture, Sports, Science and Technology, Japan.

\section{References}

1. Nakamura Y: Isolation of p53-target genes and their functional analysis. Cancer Sci 95: 7-11, 2004

2. Kamino H, Futamura M, Nakamura Y, Kitamura N, Kabu K and Arakawa H: B-cell linker protein prevents aneuploidy by inhibiting cytokinesis. Cancer Sci 99: 2444-2454, 2008.

3. Vousden KH and Lu X: Live or let die: the cell's response to p53. Nat Rev Cancer 2: 594-604, 2002.

4. Keino-Masu K, Masu M, Hinck L, Leonardo ED, Chan SS, Culotti JG and Tessier-Lavigne M: Deleted in colorectal cancer (DCC) encodes a netrin receptor. Cell 87: 175-185, 1996.

5. Leonardo ED, Hinck L, Masu M, Keino-Masu K, Ackerman SL and Tessier-Lavigne M: Vertebrate homologues of C. elegans UNC-5 are candidate netrin receptors. Nature 386: 833-838, 1997.

6. Mehlen P, Rabizadeh S, Snipas SJ, Assa-Munt N, Salvesen GS and Bredesen DE: The DCC gene product induces apoptosis by a mechanism requiring receptor proteolysis. Nature 395: 801-804, 1998.

7. Bredesen DE, Mehlen P and Rabizadeh S: Receptors that mediate cellular dependence. Cell Death Differ 12: 1031-1043, 2005. 
8. Arakawa H: p53, apoptosis and axon-guidance molecules. Cell Death Differ 12: 1057-1065, 2005.

9. Arakawa H: Netrin-1 and its receptors in tumorigenesis. Nat Rev Cancer 4: 978-987, 2004

10. Thiebault K, Mazelin L, Pays L, Llambi F, Joly MO, Scoazec JY, Saurin JC, Romeo G and Mehlen P: The netrin-1 receptors UNC5H are putative tumor suppressors controlling cell death commitment. Proc Natl Acad Sci USA 100: 4173-4178, 2003.

11. Bernet A, Mazelin L, Coissieux MM, Gadot N, Ackerman SL, Scoazec JY and Mehlen P: Inactivation of the UNC5C Netrin-1 receptor is associated with tumor progression in colorectal malignancies. Gastroenterology 133: 1840-1848, 2007.

12. Llambi F, Causeret F, Bloch-Gallego E and Mehlen P: Netrin-1 acts as a survival factor via its receptors UNC5H and DCC. EMBO J 20: 2715-2722, 2001.

13. Forcet C, Ye X, Granger L, Corset V, Shin H, Bredesen DE and Mehlen P: The dependence receptor DCC (deleted in colorectal cancer) defines an alternative mechanism for caspase activation. Proc Natl Acad Sci USA 98: 3416-3421, 2001.

14. Williams ME, Strickland P, Watanabe K and Hinck L: UNC5H1 induces apoptosis via its juxtamembrane region through an interaction with NRAGE. J Biol Chem 278: 17483-17490, 2003.

15. Tanikawa C, Matsuda K, Fukuda S, Nakamura Y and Arakawa H p53RDL1 regulates p53-dependent apoptosis. Nat Cell Biol 5 216-223, 2003.

16. Wang H, Ozaki T, Shamim Hossain M, Nakamura Y, Kamijo T, Xue $\mathrm{X}$ and Nakagawara A: A newly identified dependence receptor UNC5H4 is induced during DNA damage-mediated apoptosis and transcriptional target of tumor suppressor p53. Biochem Biophys Res Commun 370: 594-598, 2008.

17. Masuda Y, Futamura M, Kamino H, Nakamura Y, Kitamura N, Ohnishi S, Miyamoto Y, Ichikawa H, Ohta T, Ohki M, Kiyono T, Egami H, Baba $\mathrm{H}$ and Arakawa $\mathrm{H}$ : The potential role of DFNA5, a hearing impairment gene, in p53-mediated cellular response to DNA damage. J Hum Genet 51: 652-664, 2006.
18. Nakamura Y, Futamura M, Kamino H, Yoshida K, Nakamura Y and Arakawa $\mathrm{H}$ : Identification of p53-46F as a super p53 with an enhanced ability to induce p53-dependent apoptosis. Cancer Sci 97: 633-641, 2006.

19. Ishimoto O, Kawahara C, Enjo K, Obinata M, Nukiwa T and Ikawa S: Possible oncogenic potential of DeltaNp73: a newly identified isoform of human p73. Cancer Res 62: 636-641, 2002.

20. Osada M, Ohba M, Kawahara C, Ishioka C, Kanamaru R, Katoh I, Ikawa Y, Nimura Y, Nakagawara A, Obinata M and Ikawa S: Cloning and functional analysis of human p51, which structurally and functionally resembles p53. Nat Med 4: 839-843, 1998.

21. Futamura M, Kamino H, Miyamoto Y, Kitamura N, Nakamura Y, Ohnishi S, Masuda Y and Arakawa H: Possible role of semaphorin 3F, a candidate tumor suppressor gene at 3p21.3, in p53-regulated tumor angiogenesis suppression. Cancer Res 67: 1451-1460, 2007.

22. Tsukada T, Tomooka Y, Takai S, Ueda Y, Nishikawa S, Yagi T, Tokunaga T, Takeda N, Suda Y, Abe S, Matsuno I, Ikawa Y and Aizawa $S$ : Enhanced proliferative potential in culture of cells from p53-deficient mice. Oncogene 8: 3313-3322, 1993.

23. Williams ME, Lu X, McKenna WL, Washington R, Boyette A, Strickland P, Dillon A, Kaprielian Z, Tessier-Lavigne $\mathrm{M}$ and Hinck L: UNC5A promotes neuronal apoptosis during spinal cord development independent of netrin-1. Nat Neurosci 9: 996-998, 2006.

24. Thornberry NA, Rano TA, Peterson EP, Rasper DM, Timkey T, Garcia-Calvo M, Houtzager VM, Nordstrom PA, Roy S, Vaillancourt JP, Chapman KT and Nicholson DW: A combinatorial approach defines specificities of members of the caspase family and granzyme B. Functional relationships established for key mediators of apoptosis. J Biol Chem 272 : 17907-17911, 1997. 